\title{
Chronic immune barrier dysregulation among women with a history of violence victimization
}

\author{
Alison Swaims-KohImeier, ${ }^{1}$ Lisa B. Haddad, ${ }^{2}$ Tiger Zheng-Rong Li, ${ }^{3}$ Kathryn A. Brookmeyer, ${ }^{4}$ \\ James M. Baker, ${ }^{5}$ Cathy Spatz Widom, ${ }^{6}$ James C. Lamousin, ${ }^{7}$ Kai-Hua Chi, ${ }^{8}$ Cheng Y. Chen, ${ }^{8}$ \\ Ellen N. Kersh, ${ }^{8}$ Jeffrey A. Johnson, ${ }^{1}$ Melissa M. Herbst-Kralovetz, ${ }^{5}$ Matthew Hogben, ${ }^{4}$ \\ Igho Ofotokun, ${ }^{9}$ and Jacob E. KohImeier ${ }^{3}$ \\ 'Laboratory Branch, Division of HIV/AIDS Prevention, Centers for Disease Control and Prevention, Atlanta, Georgia, USA. \\ 2Department of Gynecology and Obstetrics and 'Department of Microbiology and Immunology, Emory University School \\ of Medicine, Atlanta, Georgia, USA. ${ }^{4}$ Social and Behavioral Research and Evaluation Branch, Division of STD Prevention, \\ Centers for Disease Control and Prevention, Atlanta, Georgia, USA. ${ }^{5}$ Department of Basic Medical Sciences, Department \\ of Obstetrics and Gynecology, The University of Arizona College of Medicine, Phoenix, Arizona, USA. ${ }^{6}$ Psychology \\ Department, John Jay College of Criminal Justice, and Graduate Center, City University of New York, New York, New York, \\ USA. 'Department of Mental Health, South Mississippi State Hospital, and Choices PLLC, Purvis, Mississippi, USA. \\ ${ }^{8}$ Laboratory Branch, Division of STD Prevention, Centers for Disease Control and Prevention, Atlanta, Georgia, USA. \\ ${ }^{9}$ Division of Infectious Diseases, Emory University School of Medicine, Atlanta, Ceorgia, USA.
}

\begin{abstract}
We explored the association between violence victimization and increased risk for acquiring sexually transmitted infections (STIs) in women by measuring cellular immune barrier properties from the female reproductive tract. STI-negative participants reporting repeated prior victimization occurrences through the lifetime trauma and victimization history (LTVH) instrument were more likely to exhibit alterations in barrier homeostasis and the composition of critical immune mediators irrespective of demographic parameters or presence of bacterial vaginosis. By combining cellular data with mixed-effect linear modeling, we uncovered differences in local T cells, $\mathrm{MHClI}^{+}$antigen-presenting cells, and epithelial cells indicative of altered trafficking behavior, increased immunosuppressive function, and decreased barrier integrity at sites of STI exposure that correlate most strongly with LTVH score. These data evidence a biological link between a history of violence victimization and risk of STI acquisition through immune dysregulation in the female reproductive tract.
\end{abstract}

Authorship note: ASK and LBH contributed equally to this work. I0 and JEK contributed equally to this work.

Conflict of interest: The authors have declared that no conflict of interest exists.

Copyright: (c) 2019 American Society for Clinical Investigation

Submitted: November 9, 2018

Accepted: April 11, 2019

Published: May 16, 2019.

Reference information: JCI Insight. 2019;4(10):e126097. https://doi. org/10.1172/jci.insight.126097.

\section{Introduction}

Recent statistics estimate that cases of sexually transmitted infections (STIs) are at a record high in the United States (1). STIs can lead to a range of health complications, including pelvic inflammatory disease, chronic pelvic pain, cervical cancer, and stillbirths. Identifying and understanding risk factors that increase STI susceptibility, including HIV, are critical for preventing transmission events. A growing body of literature indicates that women who have experienced events considered psychologically traumatic, such as violence victimization, are more vulnerable to STIs (2). Though prior violence victimization has been associated with sexual behaviors that may contribute to increased STI risk, the potential for traumatic experiences to have a biological impact on STI risk through long-lasting immune alterations in the female reproductive tract (FRT) has not been investigated to our knowledge.

Barrier tissue sites, including the FRT, provide the first line of defense against invading pathogens. Such peripheral tissues are not directly accessible to the circulation and hence rely on local cellular immune mediators, such as memory T cells, antigen-presenting cells (APCs), and epithelial cells, to elicit protective responses (3-6). Barrier integrity is maintained by a fine balance between tolerance to commensal organisms and the ability to mount a rapid and robust response to invading pathogens by immune cells that surveil the tissue (3-5, 7-10). Maintenance of tissue-resident immune cells under steady-state conditions is mediated in part by the expression of molecules associated with retention, 
Table 1. Demographic characteristics of the study cohort

Total cohort $(n=41) \quad$ Low-scoring group $(n=22) \quad$ High-scoring group $(n \quad P$ value

= 19)

$\begin{array}{lcccc}\text { Median number of LTVH events (range) } & 4 & 1(0-4) & 9(5-19) & 33 \\ \text { Median age } & 32 & 31.5 & 78.9 & 0.319^{\mathrm{A}} \\ \text { African American/mixed race (\%) } & 75.6 & 72.7 & 89.5 & 0.922^{\mathrm{B}} \\ \text { High school education level or higher (\%) } & 82.9 & 77.3 & 52.6 & 0.536^{\mathrm{B}} \\ \text { Annual income less than \$25,000 (\%) } & 73.2 & 90.9 & 52.6 & 0.016^{\mathrm{B}} \\ \text { Prevalence of bacterial vaginosis (\%) } & 56.1 & 59.1 & 0.920^{\mathrm{B}}\end{array}$

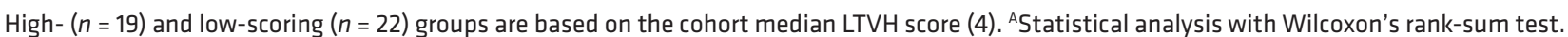
BStatistical analysis with Pearson's $\chi^{2}$ test with Yate's correction for continuity.

such as CD69 and CD103 on resident memory $\mathrm{CD}^{+} \mathrm{T}$ cells and CD69 on resident memory CD4 ${ }^{+} \mathrm{T}$ cells, and the loss of molecules associated with egress, such as CCR7 $(3-5,10,11)$. We examined key cellular immune mediators and barrier integrity at the site of STI exposure (the lower apical lumen of the FRT) to determine potential links to self-reported incidence of a lifetime trauma and victimization history (LTVH) in women (12).

\section{Results}

Demographic characteristics of this cohort are shown in Table 1. A total of 41 HIV/STI-negative women were recruited to participate in this study (For detailed eligibility criteria, see Methods). Women interested in initiating a new contraceptive were recruited into the study through community referral or self-referral and were screened for study eligibility, including medical eligibility to initiate hormonal contraception, regular menses, HIV-negative status, and no recent STIs. Then they consented for study participation and, if enrolled, were scheduled for their first study visit (baseline) approximately 3 weeks after their last menstrual period (LMP) or next menstrual period, corresponding with their luteal phase. Samples were obtained from participants before starting hormonal contraception at the relative luteal (baseline) and follicular phase (approximately 2 weeks after baseline visit and within 14 days after their LMP) of the menstrual cycle. The median age of the cohort was 32 , and $76 \%$ identified as a racial minority (African American/mixed race). The majority, $81 \%$, reported having at least a high school education, with $26 \%$ reporting incomes above the poverty line of $\$ 25,000 / y$ r. Bacterial vaginosis (BV) or prevalence of dysbiosis of vaginal flora was detected at $56 \%$ (in the US overall BV prevalence among women of reproductive age is $29 \%$, while prevalence among African American women in this group is 51\%) (13).

Psychological traumas and stressors can influence the homeostasis of the circulating T cell pool (14). To test immune fitness in the context of psychological trauma or stress from our cohort, we began by comparing circulating $\mathrm{CD}^{+} / \mathrm{CD}^{+} \mathrm{T}$ cell ratios to LTVH events (Supplemental Table 1; supplemental material available online with this article; https://doi.org/10.1172/jci.insight.126097DS1), amount of prolonged stress (Chronic Stress Scale, CSS), or participant-appraised stress (Perceived Stress Scale, PSS) reported (Figure 1A). We detected a correlation solely between the LTVH score and T cell ratio, indicating that greater LTVH events was associated with a higher $\mathrm{CD} 4^{+} / \mathrm{CD}^{+} \mathrm{T}$ cell ratio, similar to previous reports (14). To further analyze this relationship, participants were categorized according to the median LTVH score, creating a comparison of high- with low-scoring groups (Table 1, Figure 1B). Consistent with the correlation of LTVH score and $\mathrm{T}$ cell ratio, we found that although the average ratio from the low-scoring group fell into standard ranges (mean and SD: $2.27 \pm 1.06$ for women) (14), the average ratio from the high-scoring group was above the typical range (mean: 3.5 ) and significantly higher in comparison with the low-scoring group.

To evaluate whether LTVH events experienced in this cohort might also signify $\mathrm{T}$ cell irregularities at sites of STI exposure in the periphery, we performed a broad characterization of immune cells localized at the lumen of the FRT using an enhanced atraumatic cervical vaginal lavage technique (ref. 11 and Figure 2). Cell enrichment from lavages generated a consistent range of epithelial and leukocyte populations (including APCs and T cells) among the high- and low-scoring groups (Figure 2A). Focusing on $\mathrm{MHCII}^{+}$ APC populations (Supplemental Figure 1), which regulate FRT T cell function (3), we measured CD103, 
A
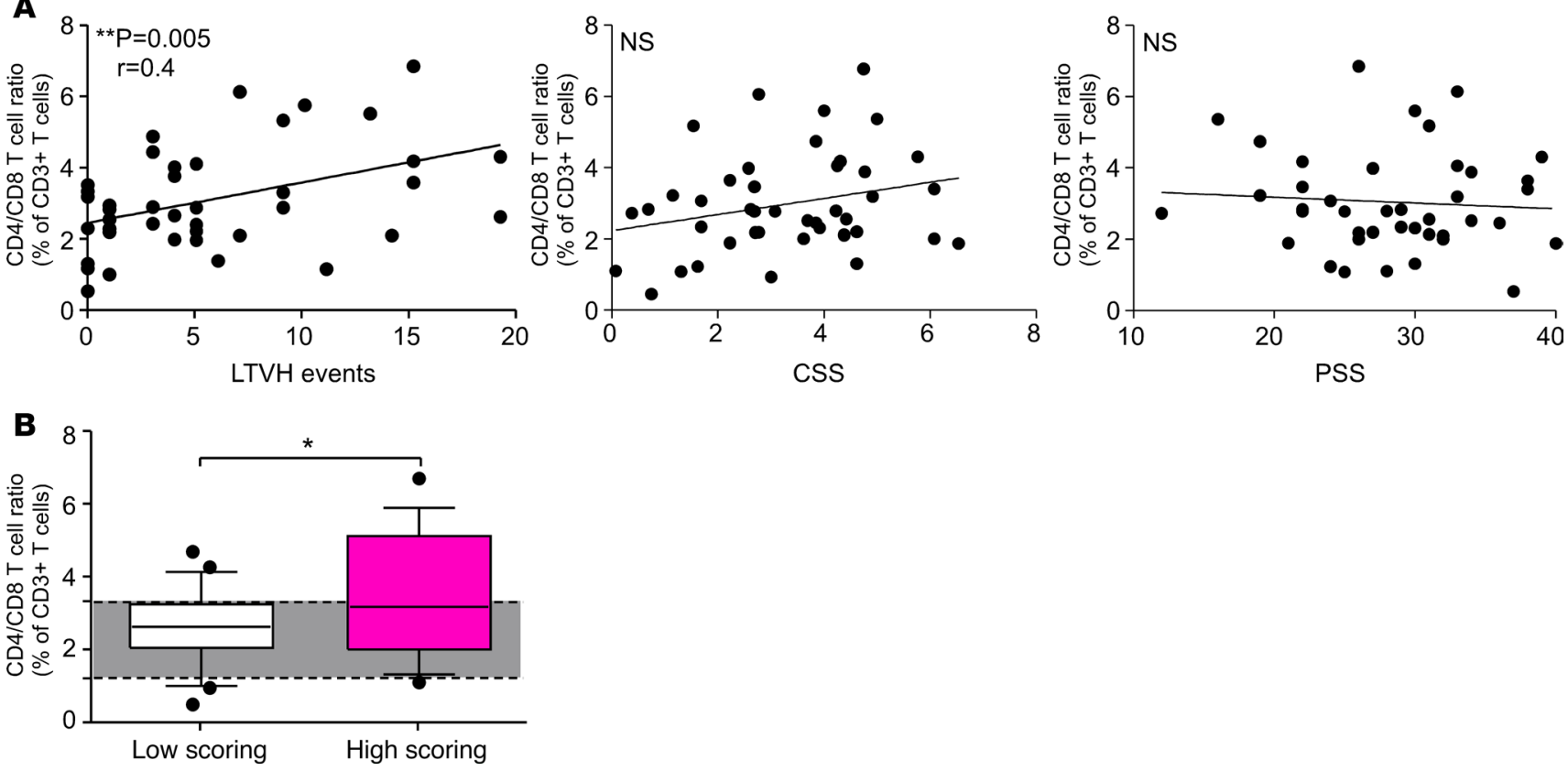

Figure 1. The LTVH score is associated with T cell ratios in PBMC. (A) The CD4 $4^{+} / \mathrm{CD} 8^{+}$T cell ratio measured from PBMCs $(n=41)$ and correlated to LTVH score, Chronic Stress Scale (CSS) score, or Perceived Stress Scale (PSS) score from participants. Significance calculated by Pearson's correlation $(r=0.06$, and $P=0.721)$. (B) Box-and-whisker plot of the $C D 4^{+} / C D 8^{+} T$ cell ratio compared by participants grouped by either high or low LTVH events reported. The high LTVH-scoring group exhibited a higher mean T cell ratio $\left({ }^{*} P=0.046\right)$ compared with the low LTVH-scoring group. Significance calculated by an unpaired, 2-tailed Student's $t$ test. Gray shading represents the reference range of PBMC T cell ratios for women. Box-and-whisker plot depicts the interquartile range with median bar and with whiskers depicting minimum and maximum values.

an integrin defining tolerogenic dendritic cells (DCs) that can induce immunosuppressive regulatory $\mathrm{T}$ cell (Treg) differentiation (15-17); CCR5, a chemokine receptor important for APCs' peripheral recruitment (18); and CCR7, a chemokine receptor important for trafficking from peripheral tissues into afferent lymphatics (18). APC populations from the high-scoring group expressed increased CCR5 and CD103 frequency, while no difference was detected with CCR7 (Figure 2B).

To evaluate lower FRT epithelial cells, we performed quantitative reverse transcription PCR to measure gene transcripts of proteins involved in barrier integrity and inflammation (Figure 2C). Within the high-scoring group, we found lower expression of genes that function in maintaining epithelial cell barrier integrity: $L C E 3 D, O C L N$, and $A L O X 12$. Regarding genes involved in epithelial cell-mediated immune protection, within the high-scoring group we found decreased transcript expression of TNFA.

To measure regional barrier properties of FRT T cell populations, $\mathrm{CD}^{+}$and $\mathrm{CD}^{+} \mathrm{T}$ cells (Figure 2D) were characterized for expression of tissue residence or recirculation markers. Local retention (CD69 and CD103) or egress (CCR7) presentation $(3,5)$ by $\mathrm{T}$ cells revealed substantial differences between groups (Figure 2D). Although the majority of FRT T cells expressed CD69 and an increased frequency of CD103 (contrasting with matched circulating cells, Supplemental Figure 2), T cell populations from the high-scoring group exhibited a decreased frequency of CD69 expression. This corresponded to increased CCR7 frequency among $\mathrm{T}$ cells from the high-scoring group, particularly within $\mathrm{CD} 4^{+} \mathrm{T}$ cells. Coexpression analysis of CCR7, CD69, and CD103 showed that although T cells from the low-scoring group samples were principally expressing tissue retention profiles, consistent with $\mathrm{T}$ cell properties at an immune-restricted tissue site (19), $\mathrm{CD}^{+} \mathrm{T}$ cells from the high-scoring group samples exhibited predominantly egress profiles $\left(\mathrm{CCR} 7^{+} \mathrm{CD} 69^{-} \mathrm{CD}^{-103}{ }^{-}\right.$) (Figure $2 \mathrm{D}$, pie charts). $\mathrm{CD} 8^{+} \mathrm{T}$ cells from the high-scoring group did exhibit increased egress properties; however, they were predominantly expressing greater retention characteristics, similar to $\mathrm{CD}^{+} \mathrm{T}$ cells from the low-scoring group samples.

Because BV or phase of the menstrual cycle can alter FRT immune cell populations, we asked whether these factors might have influenced or accounted for the differences observed within the high-scoring group $(21,22)$. To address these questions, we reanalyzed immune measures using only BV-negative samples (Supplemental Figure 3) or stratified our analysis based on relative phase of the menstrual cycle when samples 
A

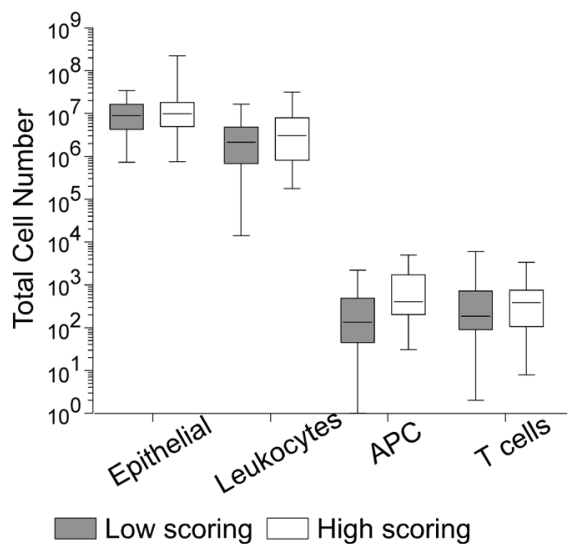

B

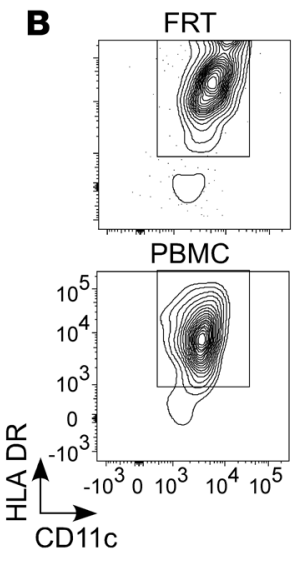

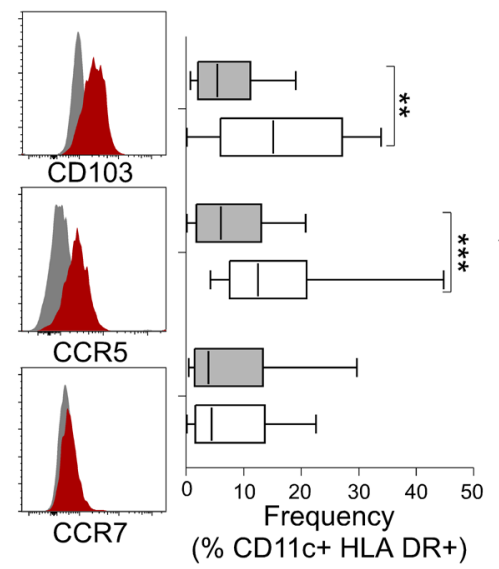

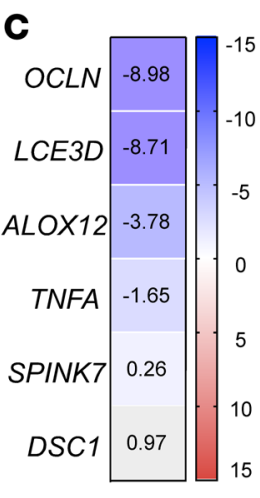

D
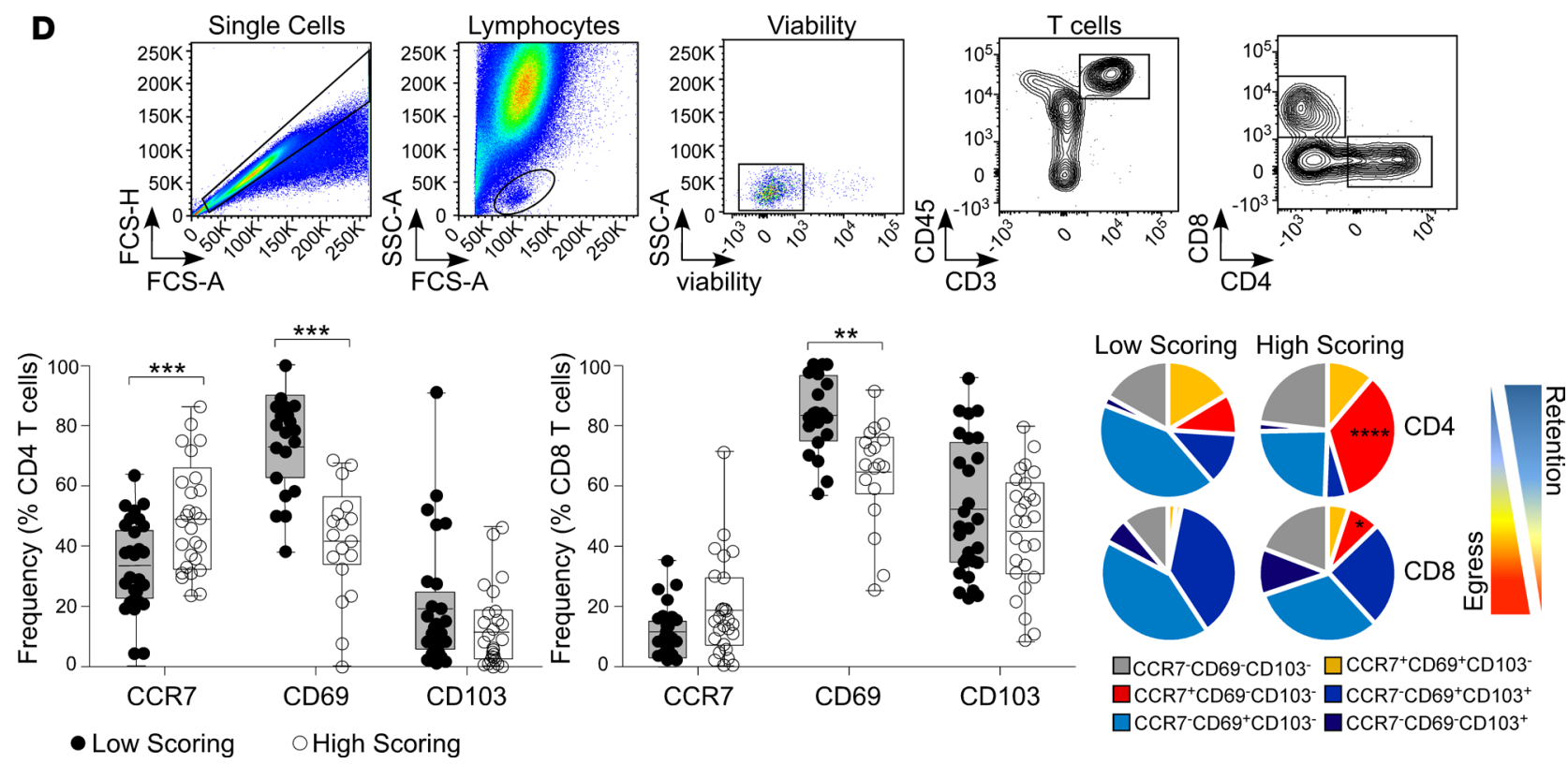

$\square \mathrm{CCR} 7{ }^{-C D 69-C D 103-} \square \mathrm{CCR} 7^{+} \mathrm{CD} 69^{+} \mathrm{CD} 103^{-}$

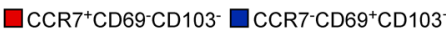
$\square$ CCR7 $-\mathrm{CD} 69^{+} \mathrm{CD} 103-\mathrm{CCR} 7-\mathrm{CD} 69^{-\mathrm{CD}} 103^{+}$

Figure 2. Prior trauma and victimization experiences reported are associated with phenotypic deviations of critical cellular immune mediators at the FRT barrier. (A) Cell numbers from participant samples $(n=66)$ with low (gray box and whiskers) compared with high LTVH event scores (white box and whiskers) (box depicting the interquartile range with median bar and whiskers depicting minimum and maximum values). (B) Representative flow plots (left) depicting APC gating from viable leukocytes from FRT (top) and matched PBMCs (bottom). Histograms illustrating CD103, CCR5, and CCR7 expression measurement from APCs (labeled center); FRT samples (red overlay) are compared with matched PBMCs (gray overlay). Frequency of chemokine receptor or integrin APC expression ( $n=55$ ) from high- (white box and whiskers) and low-scoring (gray box and whiskers) participant samples (right) (box depicting the interquartile range with median bar and whiskers depicting minimum and maximum values). (C) Quantitative reverse transcription PCR heatmap depicting fold change in epithelial gene transcript measurement from high-compared with low-scoring participant samples. (D) Representative gating strategy for T cell characterization (top). Viable T cells $(n=54)$ are distinguished for CD4 and CD8 expression. CCR7, CD69, and CD103 expression frequency of $\mathrm{CD}^{+}$and $\mathrm{CD} 8^{+} \mathrm{T}$ cells from high-scoring (white-circle background box and whiskers) and low-scoring (black-circle background gray box and whiskers) samples (box depicting the interquartile range with median bar and whiskers depicting minimum and maximum values) (bottom left). Boolean analysis of CCR7, CD69, and CD103 coexpression frequency from CD4+ and CD8 ${ }^{+}$T cells (bottom right). Significance calculated by an unpaired, 2-tailed Student's $t$ test. ${ }^{* *} P<0.01 ;{ }^{* *} P<0.001 ;{ }^{* * *} P<0.0001$.

were collected (Figures 3 and 4). Using samples that were determined to be BV negative (samples scored as intermediate were also excluded), we found the same immune patterns when comparing high- and low-scoring LTVH groups (Supplemental Figure 3). To determine potential impacts of the menstrual cycle, we analyzed longitudinal cellular barrier measurements from samples collected at the luteal or follicular phase. Similar to the combined data, we observed no significant changes in the number of immune cells (Figure 3) between high- and low-scoring LTVH groups at either the follicular or luteal phases of the menstrual cycle. In addition, the significant alterations in APC subsets (Figure 3 ) and $\mathrm{CD} 4^{+}$and $\mathrm{CD} 8^{+} \mathrm{T}$ cell subsets (Figure 4) observed 
A
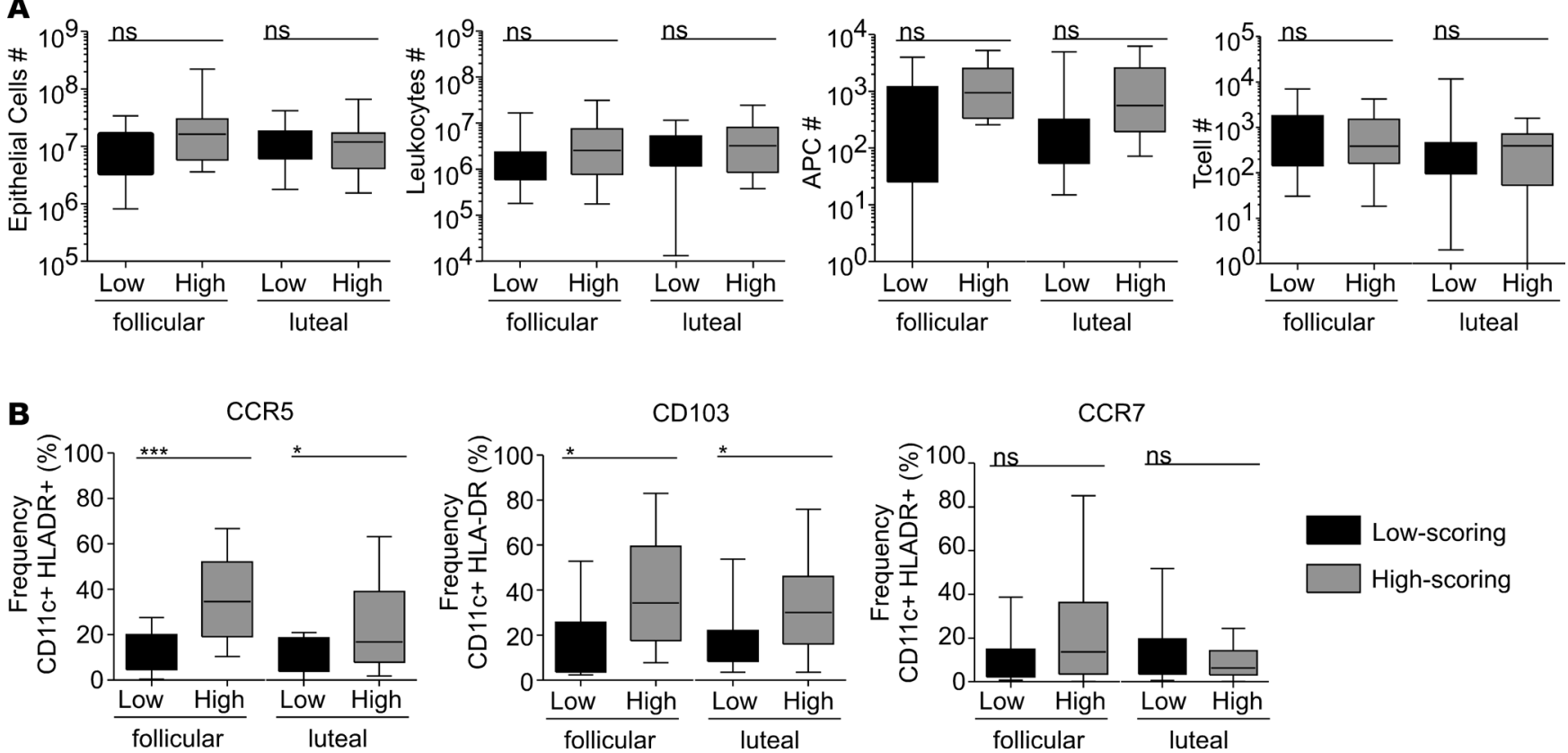

Figure 3. Stage of the menstrual cycle does not alter the impact of LTVH score on FRT immune cell numbers or the distribution of APC subsets. (A) Cell abundance of epithelial cells, leukocytes, APCs, and T cells detected from vaginal lavage collections taken at the relative luteal or follicular phase of the menstrual cycle and compared with low or high LTVH scores $(n=69)$. Longitudinal comparisons at the follicular or luteal phase of the menstrual cycle among either low-scoring (black box and whiskers) or high-scoring groups (gray box and whiskers) were analyzed using a paired Student's $t$ test. Comparisons between low-scoring and high-scoring measures at either the follicular or luteal phase of the menstrual cycle were analyzed using an unpaired, 2-tailed Student's $t$ test. (B) Extracellular expression frequency of CCR5, CD103, and CCR7 from CD11c ${ }^{+} H L A-D R^{+}$APC populations $(n=64)$ analyzed both longitudinally across the course of 1 menstrual cycle or by comparing group scores at either the follicular or luteal phase. Statistics calculated similarly to $\mathbf{A}$ (box depicting the interquartile range with median bar and whiskers depicting minimum and maximum values). ${ }^{*} P<0.05 ;{ }^{* *} P<0.001$.

in the high-scoring LTVH group were present at both the follicular and luteal phases. We also performed a combined analysis of LTVH score and phase of the menstrual cycle to investigate the contribution of these parameters to the measured immune parameters (Table 2). The differences in APC, CD4 ${ }^{+} \mathrm{T}$ cell, and CD8 T cell subsets observed were most strongly associated with LTVH score but not phase of the menstrual cycle. Together, these data indicate that the altered immune barrier properties detected in the high-scoring LTVH group were not associated with or modulated by the menstrual cycle.

Comparisons in the context of the menstrual cycle or BV status showed consistent immune dysregulation among high LTVH- compared with low LTVH-scoring samples and indicated that neither presence of BV nor phase of the menstrual cycle accounted for differences among these groups. To further validate whether the cellular FRT immune barrier differences observed were attributable primarily to overall LTVH score, we performed mixed-effect linear regression modeling (Table 3) focusing on FRT immune measures with the presence of BV or phase of the menstrual cycle as potential confounders. In agreement with our experimental data, these results showed that the FRT immune barrier variations detected from high-scoring samples were not dependent on the presence of BV or influenced by the menstrual cycle but instead were most strongly associated with LTVH score.

Early-life adversity is linked to a higher risk of developing immune-based diseases and chronic inflammation (22). To evaluate the age of onset and LTVH event scores within participants, we first examined the low- and high-scoring LTVH groups according to reported age of the first LTVH event. We found that the high-scoring LTVH group participants were more likely to experience their first LTVH event significantly earlier in life than participants in the low-scoring LTVH group who had reported at least 1 LTVH event $(P<0.0001)$ (Supplemental Table 2 and Supplemental Figure 4). To analyze the immune impact of LTVH onset occurring in childhood, we stratified the demographic parameters and immune data by participants reporting LTVH events occurring at or before 12 years of age (early onset) and compared them with participants reporting LTVH events occurring only after 
A

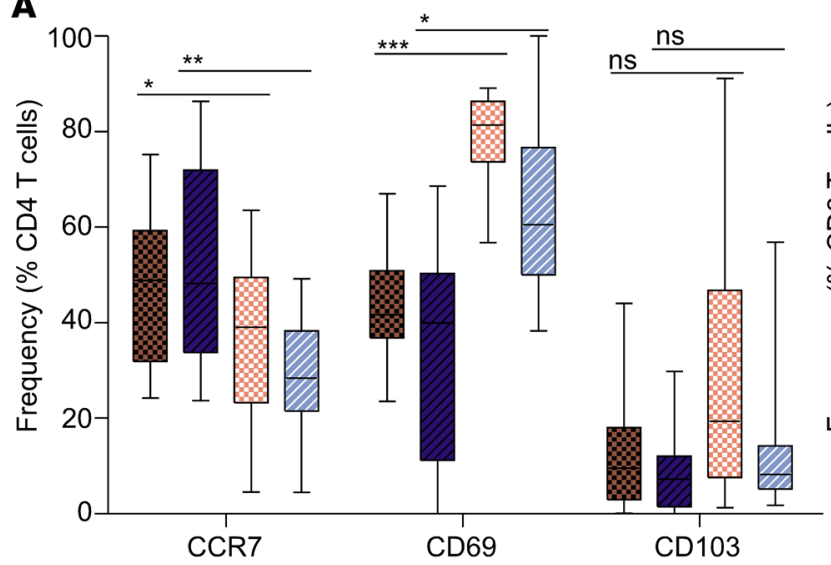

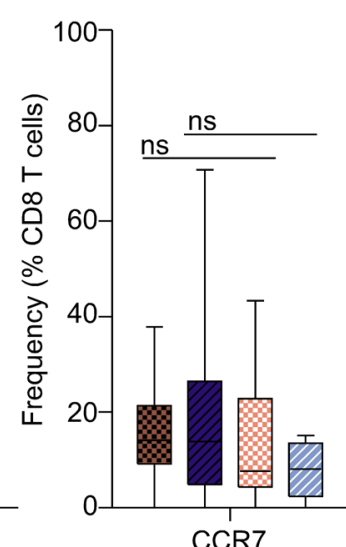

CCR7
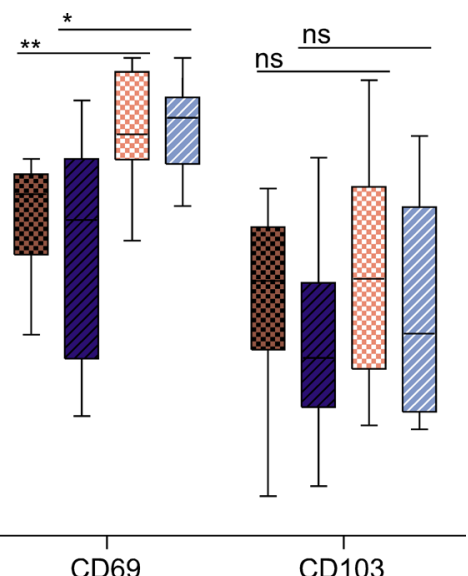

88High-scoring Luteal

High-scoring Follicular

3 Low-scoring Luteal ULLLow-scoring Follicular

B
CD4 T cells

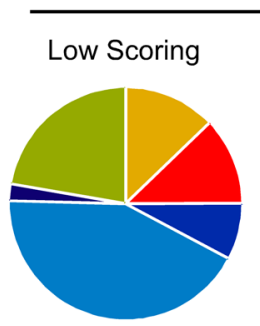

High Scoring

Follicular
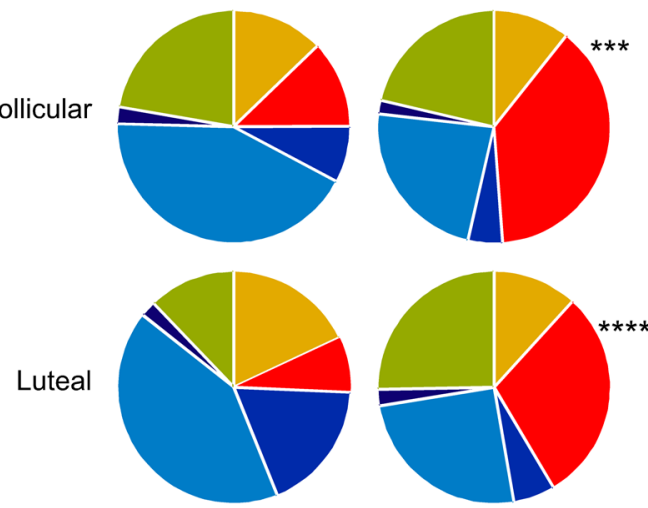

CD8 T cells

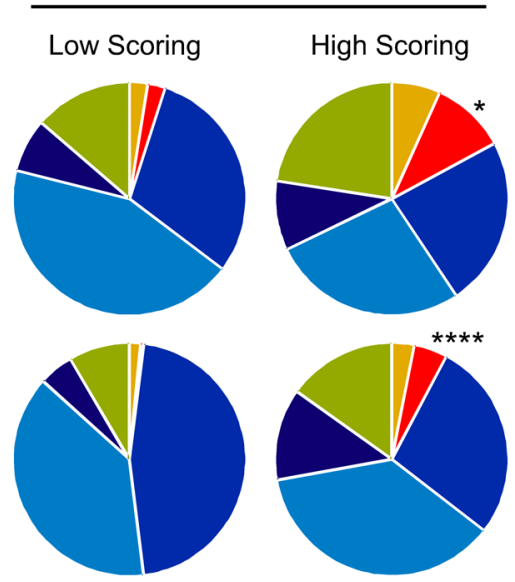

\section{$\square$ CCR7-CD69-CD103- $\square \mathrm{CCR7-CD69+CD103^{- }}$ $\square \mathrm{CCR}^{+}{ }^{+} \mathrm{CD}_{69}{ }^{+} \mathrm{CD} 103^{-}-\mathrm{CCR} 7^{-} \mathrm{CD} 69^{+} \mathrm{CD} 103^{+}$ 口CCR7 ${ }^{+}{ }^{C D 69-C D 103-}$ - CCR7-CD69-CD103 ${ }^{+}$}

Figure 4. Stage of the menstrual cycle does not alter the impact of LTVH score on the dysregulation of memory T subsets in the FRT. (A) CCR7, CD69, and $\mathrm{CD} 103$ expression frequency of $\mathrm{CD} 4^{+}$and $\mathrm{CD} 8^{+} \mathrm{T}$ cells from high- and low-scoring samples compared based on collections taken at the relative luteal or follicular phase of the menstrual cycle (samples depicted as box and whiskers, with box depicting the interquartile range with median bar and whiskers depicting minimum and maximum values; $n=54$ ). Statistics calculated from longitudinal comparisons were analyzed using an unpaired 2-tailed Student's $t$ test, while samples compared based on high- or low-scoring LTVH were analyzed using an unpaired, 2-tailed Student's $t$ test. (B) Boolean analysis of CCR7, CD69, and CD103 coexpression frequency from CD4 ${ }^{+}$and CD8 ${ }^{+} T$ cells measured from vaginal lavage collections taken at the relative luteal or follicular phase of the menstrual cycle and compared by low or high LTVH scores. ${ }^{*} P<0.05 ;{ }^{* *} P<0.01 ;{ }^{* *} P<0.001 ;{ }^{* * *} P<0.0001$. Asterisks represent a significant increase in CCR7 ${ }^{+}$CD69- $C D 103^{-} \mathrm{T}$ cell populations (shown as red shading) in high-scoring compared with low-scoring groups at indicated phases of the menstrual cycle.

12 years of age (Supplemental Tables 3 and 4). As shown (Supplemental Table 3), demographics and prevalence of BV were not significantly different among early- and late-onset LTVH groups. Hence, after controlling for the BV score and phase of menstrual cycle in the mixed-effect linear model (Supplemental Table 4), the incorporation of the LTVH onset indicator improved model fitting only for the $\mathrm{CD}^{2} 9^{+} \mathrm{CD}^{+} \mathrm{T}$ cell and $\mathrm{CD} 69^{+} \mathrm{CD}^{+} \mathrm{T}$ cell immune profiles; however, the effect was not significant ( $P=0.24$ and $P=0.38$, respectively). The negative impact of LTVH score on CD69 ${ }^{+} \mathrm{CD} 4^{+} \mathrm{T}$ cells remained significant when LTVH onset is taken into account $(P=0.0006$ vs. $P=0.0048)$, while the impact on $\mathrm{CD} 69^{+} \mathrm{CD} 8^{+} \mathrm{T}$ cells was no longer significant $(P=0.017$ vs. $P=0.11)$. Therefore, although age of onset and overall LTVH score were closely linked, the modeling data support our overall conclusion that total LTVH score, but not age of initial LTVH onset, is the primary factor associated with immune dysregulation in the FRT. 
Table 2. Two-way ANOVA for repeated measures assessing the significance of main effects of LTVH score and phase of the menstrual cycle on altered immune barrier properties

\begin{tabular}{|c|c|c|}
\hline Immune parameter & LTVH score $\boldsymbol{P}$ values ${ }^{A}$ & MC phase $\boldsymbol{P}$ values \\
\hline APC number & 0.180 & 0.681 \\
\hline CCR5+ APCs & $<0.0001$ & 0.686 \\
\hline $\mathrm{CD} 103^{+} \mathrm{APCs}$ & 0.0009 & 0.636 \\
\hline $\mathrm{CCR}^{+} \mathrm{CD}^{+} \mathrm{T}$ cells & 0.0023 & 0.715 \\
\hline $\mathrm{CD}^{\circ}{ }^{+} \mathrm{CD}^{+} \mathrm{T}$ cells & $<0.0001$ & 0.058 \\
\hline $\mathrm{CD}^{2} 3^{+} \mathrm{CD}^{+} \mathrm{T}$ cells & 0.073 & 0.273 \\
\hline CCR7+CD8+ T cells & 0.143 & 0.463 \\
\hline CD69+CD8+ T cells & 0.0029 & 0.479 \\
\hline $\mathrm{CD}{ }^{\circ} 3^{+} \mathrm{CD}^{+} \mathrm{T}$ cells & 0.264 & 0.161 \\
\hline Epithelial cell numbers & 0.303 & 0.289 \\
\hline
\end{tabular}

\section{Discussion}

Though the relationship between the central nervous system and the immune system is not yet fully explored, prior studies show that psychological stressors and traumas can affect immune responses and lead to increased susceptibility to various infections (14, 23-25). Violence victimization has been linked to an increased risk of STI acquisition (2), but long-term biological effects that may influence this increased risk have thus far not been extensively investigated. To our knowledge, this study is the first to characterize immune barrier properties in relation to past experiences of trauma and violence victimization. These data suggest that women who have endured greater violence victimization and psychologically traumatic events are more likely to experience alterations in immune barrier composition in the FRT.

Table 3. Mixed-effect linear regression modeling of parameters influencing immune cell composition in the FRT

\begin{tabular}{|c|c|c|}
\hline Variables & Estimate of regression coefficient (SEM) & $P$ value \\
\hline \multicolumn{3}{|l|}{$\mathrm{CCR}^{+}$APCs $(n=42)$} \\
\hline LTVH (high scoring) & $25.5(5.3)$ & 0.0003 \\
\hline BV (positive) & $-5.9(5.2)$ & 0.2822 \\
\hline MC phase (luteal) & $-6.1(4.1)$ & 0.1540 \\
\hline \multicolumn{3}{|l|}{$\mathrm{CD}^{\circ} 3^{+} \mathrm{APCs}(n=42)$} \\
\hline LTVH (high scoring) & $30.0(7.9)$ & 0.0020 \\
\hline BV (positive) & $0.3(7.9)$ & 0.9683 \\
\hline MC phase (luteal) & $5.0(4.4)$ & 0.2701 \\
\hline \multicolumn{3}{|l|}{ CCR7+CD4+ ${ }^{+}$cells $(n=43)$} \\
\hline LTVH (high scoring) & $16.8(4.9)$ & 0.0042 \\
\hline BV (positive) & $9.7(4.9)$ & 0.0660 \\
\hline MC phase (luteal) & $-1.8(4.7)$ & 0.7126 \\
\hline \multicolumn{3}{|l|}{ CD69 + CD4 $^{+} T$ cells $(n=30)$} \\
\hline LTVH (high scoring) & $-38.2(5.3)$ & $<0.0001$ \\
\hline BV (positive) & $-21.8(5.2)$ & 0.0024 \\
\hline MC phase (luteal) & $10.7(5.0)$ & 0.0595 \\
\hline \multicolumn{3}{|l|}{ CD69 + CD8 $^{+} T$ cells $(n=30)$} \\
\hline LTVH (high scoring) & $-22.0(5.8)$ & 0.0044 \\
\hline BV (positive) & $-16.1(5.8)$ & 0.0212 \\
\hline MC phase (luteal) & $6.4(5.5)$ & 0.2727 \\
\hline
\end{tabular}

LTVH (high or low scoring), presence of BV (positive or negative), and menstrual phases (luteal or follicular) were analyzed as fixed effects. For each group, $n$ values are indicated. Bold text indicates statistical significance. 
Cellular characterization at the FRT lumen in the context of reported LTVH displayed patterns indicative of local immune suppression, increased $\mathrm{T}$ cell trafficking from the circulation, and decreased epithelial barrier integrity. $\mathrm{MHCII}^{+} \mathrm{APC}$ populations expressed properties associated with increased peripheral tissue trafficking and increased tolerogenic DC subsets, which can promote T cell-mediated immune suppression through directing Treg versus effector $\mathrm{T}$ cell differentiation (15-17). Corresponding to this observation, $\mathrm{CD}^{+} \mathrm{T}$ cells exhibited greater CCR7 expression, which identifies T cells in the skin and HIV target cells in the lower FRT (migratory or recirculating memory T cells) that can reenter afferent lymphatic circulation $(11,26)$. A marked decrease of $\mathrm{CD}^{+}$and $\mathrm{CD} 8^{+} \mathrm{T}$ cell populations expressing tissue retention characteristics was observed, consistent with recent immigration of $\mathrm{T}$ cells from the circulation that are not specialized to function in tissue immune surveillance $(10,27)$. Gene expression measurement in epithelial cells indicates reduced barrier integrity in the high-scoring group because of decreased expression of genes such as $O C L N$ and $L C E 3 D$. Although $L C E 3 D$ downregulation has been associated with vaginal barrier disruption (28), OCLN downregulation has been previously observed in response to psychological stress (29). In addition to reduced barrier integrity, lower TNFA expression was detected, indicating a reduced presence of local proinflammatory cytokines involved in epithelial cell-mediated protection from STIs (30).

Together, these findings describe a local environment at the FRT interface more permissive to infection via increased opportunities for pathogens to traverse the epithelia, less robust local immune surveillance, and attenuated early immune responses. Though BV, stage of the menstrual cycle, and chronic stress have all been shown to influence immune responses (21-23), we did not find confounding interactions with LTVH score and cellular barrier measurements. Because BV likely promotes a local proinflammatory immune environment (31) and LTVH-related immune differences were consistent at either the luteal or follicular phase of the menstrual cycle, this raises the possibility of unique mechanisms of immune-mediated STI susceptibility contributed by an LTVH. It is interesting to note that the high-scoring LTVH group participants were also more likely to experience their first LTVH events in childhood ( $\leq 12$ years of age). Although age of onset did not confound immune barrier dysregulation detected with the LTVH score, our findings evidence a link between early-life trauma and likelihood of repeated LTVH events in the context of detected immune alterations. Though additional key demographics obtained in this focused cohort did not influence our findings, noninclusion of a broader range of potential confounders may have augmented the associations observed. Thus, future studies involving broader participant groups, greater longitudinal sampling, and additional sociobehavioral characteristics, and further discerning the nature of psychological traumas experienced, with immune-based measures are critical to better understanding the immunologic link between psychological traumas and STI risk.

As demonstrated by the results of this study, physician inquiries into lifetime violence and trauma as part of a comprehensive history can assist health care providers in interpreting health complications and modifying the individual's risk of STIs, including HIV. These findings support current Centers for Disease Control and Prevention (CDC) guidance to gather comprehensive sexual histories, with the addition of a LTVH score, to better assist populations vulnerable to STI acquisition (1).

\section{Methods}

Study participants. This evaluation included 41 women participating in a longitudinal study assessing the effects of hormonal contraception on HIV biomarkers in Atlanta, Georgia (2015-2017). Women interested in initiating a new contraceptive were recruited into the study through community referral or self-referral and were screened for study eligibility, including HIV-negative status (OraQuick swab) and no recent STIs (self-report and later tested by reverse transcription PCR described below). Then they consented for study participation and, if enrolled, were scheduled for their first study visit (baseline) approximately 3 weeks after their LMP next menstrual period, corresponding with their luteal phase. Eligibility for participation required that women were aged 18-45 years, exhibiting normal menses (22- to 35-day cycles for at least 3 cycles), not using any hormonal contraception method or intrauterine device for at least 6 months prior, not pregnant for at least 3 months prior, not breastfeeding, and not experiencing symptomatic vaginal infection or genital ulcer disease at screening. Participants did not have a history of loop electrosurgical excision procedure, conization, or cryosurgery in the previous year. Participants were further screened for medical eligibility based on CDC medical eligibility criteria for contraceptive use and clinical judgment (32). A questionnaire including sociodemographic and behavioral characteristics was completed along with a cervicovaginal swab followed by a cervicovaginal lavage (CVL) (11) and blood specimen collected from women at each visit. 
LTVH cohort description. Among all participants the median LTVH score was 4 . Based upon the cohort median LTVH score, we stratified data into low-scoring (LTVH events less than or equal to $4, n=22$ ) or high-scoring (LTVH events more than $4, n=19$ ) groups (Table 1). Among the high-scoring group, the median LTVH number was 9 (IQR: 5-19) whereas for the low-scoring group, the median LTVH score was 1 (IQR: $0-4)$. The medians of age, composition of races, and education levels were not significantly different between the 2 groups. However $91 \%$ of low-scoring participants reported living below the poverty line (annual income $<\$ 25,000)$, significantly higher than that of high-scoring participants $(P=0.016)$. Lower income was not associated with immune dysregulation properties; thus, we did not control for covariates in further analysis. Total BV prevalence was 56\%, with 53\% in the high-scoring group and 59\% in the low-scoring group.

Psychological stress or trauma scoring. The validated LTVH instrument was used to assess lifetime trauma and violence victimization history as previously described (12) along with the CSS and the PSS. The LTVH, CSS, and PSS were administered as part of a questionnaire at the initial study visit. Participants were asked a series of questions concerning their exposure to 30 traumas and violence victimization experiences (including general traumas, physical assault/abuse, sexual assault/abuse, kidnapping/stalking, family/friend murdered or committed suicide, witnessed trauma to someone else, and crime victimizations), age at the time of onset of the experience, degree of danger and fear experienced, and frequency of each experience (Supplemental Table 1). Participant answers were compiled and scored as number of LTVH events. Reported trauma and victimization histories were compared using the median score of all participants, with individuals categorized as low LTVH for those at or below the median and high LTVH for those above median score. The CSS questionnaire was administered to assess prolonged emotional pressure from a list of 51 items about common life conditions and situations (e.g., financial issues, work, love and marriage, family and children, social life). Answers were compiled from participant responses of not true (score 0), somewhat true (score 1), or very true (score 2). The PSS questionnaire was administered to measure the degree participants appraised a situation as stressful and included 10 questions about the participant's feelings and thoughts during the past month (33). Answers were compiled from response options for each feeling or thought by frequency with which it occurred: 0 = never; 1 = almost never; 2 = sometimes; 3 = fairly often; 4 = very often.

STI and BV testing. Cervicovaginal swabs were collected by a trained provider and tested for STIs; DNA was extracted using DrySwab (Lakewood Biochemical Company) using the Qiagen DNA Mini kit and used to amplify targets from Neisseria gonorrhoeae, Chlamydia trachomatis, Mycoplasma genitalium, Trichomoniasis vaginalis (34), and herpes simplex virus types 1 and 2 (35), using 2 real-time duplex PCR assays and Qiagen Rotor-Gene Q real-time PCR instrument. Qiagen Rotor-Gene Q series software was used to analyze data. Cell smears from CVL were used for Gram stains and assigned Nugent scores. The number of Lactobacillus, Gardnerella, Bacteroides, or curved gram-indeterminate rods were used to calculate Nugent scores from CVL cell smears. Total scores above 6 were consistent with BV, and scores below 4 were considered BV negative. Intermediate scores of 4 to 6 were considered indeterminate.

Cell isolation and characterization. Blood was collected in 8-ml, sodium citrate-containing CPT tubes (BD Biosciences) and separated into plasma and PBMCs by centrifugation. Lavage specimens were enriched for leukocytes by density centrifugation as previously described (11); epithelial cells were collected at the relative luteal phase from the top fraction of the density gradient. Lavage samples with visible blood or detectable naive $\mathrm{T}$ cell populations $\left(\mathrm{CCR} 7^{\mathrm{hi}} \mathrm{CD} 45 \mathrm{RA} \mathrm{A}^{\text {hi }}\right)$ were considered contaminated with cells from circulation and excluded from characterization. Leukocytes were stained for viability using Zombie Yellow Fixable Viability Kit (BioLegend), then incubated with anti-CD16/32 Fc block (BioXCell). Cells were stained with the following fluorochrome-conjugated antibodies: CD3 (V450, UCHT1), CD4 (Alexa Fluor 700, RPA-T4), CD8 (BV510, RPA-T8), CCR7 (PE-CF594, 150503), CCR5 (PE, 3A9), and CD103 (FITC, Ber-ACT8) from BD Biosciences and CD11c (BV711, 3.9), CD45 (BV650, H130), CD45RA (BV605, HI100), CD66b (Alexa Fluor 647, G10F5), CD69 (APC/Fire 750, FN50), and HLA-DR (BV785, L243) from BioLegend. Samples were run on a BD Biosciences LSRII flow cytometer. Data were acquired using FACSDIVA software (BD Biosciences) and analyzed using FlowJo software (Tree Star, Inc.).

Reverse transcription PCR. Epithelial cell pellets from BV-negative samples were transported in RNAlater (Thermo Fisher Scientific) and stored at $-80^{\circ} \mathrm{C}$. RNA was extracted using the Zymo Mini RNA (Plus) isolation kit (Zymo Research) with proteinase K treatment. We used 160 ng of RNA for cDNA synthesis using the iScript cDNA synthesis kit (Bio-Rad). Quantitative PCR was completed for the following genes: occludin, desmocollin-1, serine peptidase inhibitor kasal type 7, late cornified envelope 3D, TNF- $\alpha$, and arachidonate 12-lipoxygenase. Values were normalized to the housekeeping gene GAPDH. We calculated $\Delta \Delta \mathrm{CT}$ 
fold change using the averages of the control group compared with the LTVH group. The following primers were used: GAPDH (forward primer) TCATGACCACAGTCCATGCCA, (reverse primer) CCCTGTTGCTGTAGCCAAATT; DSC1 (forward primer) TGCCAAACACTCCTCACTCAAA, (reverse primer) TTCAATGGCTTGACAACACACA; ALOX12 (forward primer) TATCTTGAATTTCATGCTTTCCTAAAGTC, (reverse primer) GCGGTCTGCAAAGCGTAAAA; LCE3D (forward primer) CTCTGCACCTGGACAACTCA, (reverse primer) CACTTGGGTGAGGGACACTT; SPINK7 (forward primer) ATGAAGATCACTGGGGGTCTCCT, (reverse primer) TTAGCAACTTCCATCGTGAAGA; TNFA (forward primer) CTCAACCTCTTCTGGCTCAAA, (reverse primer) GTCAGGGATCAAAGCTGTAGG; OCLN (forward primer) CCCCATCTGACTATGTGGAA, (reverse primer) CCGCTTGTCATTCACTTTGCC.

Statistics. Statistical analyses were performed in Prism 5 software (GraphPad) and SAS 9.4. Unless otherwise noted, 2-tailed Student's $t$ test for independent samples was used to determine significance $\left({ }^{*} P\right.$ $\left.<0.05 ;{ }^{*} P<0.01 ;{ }^{* *} P<0.001 ;{ }^{* * * *} P<0.0001\right)$. Statistical tests for demographics and immune measures are noted in the table and figure legends. Two-way ANOVA for repeated measures was conducted to compare the main effects of LTVH scoring (high or low), phases of menstrual cycle (luteal or follicular), and their statistical interaction. To control for potential confounding parameters and account for the between-subject variation, we performed mixed-effect linear regression with LTVH scoring (high or low), BV (positive or negative), and phases of menstrual cycle (luteal or follicular) as fixed effects and subjects as random effect. Subjects with only 1 visit or changed BV status were excluded from the model fitting.

Study approval. Protocols were approved by the Emory University Institutional Review Board and the Grady Research Oversight Committee. All participants were given and signed an informed consent document.

\section{Author contributions}

ASK and LBH performed experiments, analyzed data, and interpreted data. TZRL performed statistical and modeling analyses. JMB performed quantitative reverse transcription PCR experiments. JMB and MMHK analyzed and interpreted quantitative reverse transcription PCR data. KHC performed STI testing. KHC and CYC analyzed and interpreted STI results. KAB, MH, CSW, JCL, CYC, ENK, and JAJ provided critical advice and helped with the interpretation and critical discussion of the results. $\mathrm{LBH}$, ASK, IO, and JEK designed the study and performed analyses. ASK and JEK wrote the manuscript, with contributions from LBH, TZRL, KAB, JMB, CSW, JCL, KHC, CYC, ENK, JAJ, MMHK, MH, and IO.

\section{Acknowledgments}

We thank the study participants and colleagues who contributed to study implementation. We thank the Atlanta Clinical and Translational Research Institute Clinical Research Network (Grady site), the KL2-Mentored Clinical and Translational Research Program, the Grady Infectious Disease Program, and the Emory Center for AIDS Research Clinical Core. At the CDC we acknowledge Clyde Hart, Richard Haaland, and Tammy Evans-Strickfaden for assistance overlapping from Hormonal Contraception study design and implementation and L. Davis Lupo for technical expertise with CVL processing. The findings and conclusions in this report are those of the authors and do not necessarily represent the views of the US CDC or the US Department of Health and Human Services. This study was supported by the US CDC and Emory University and in part by NIH grants R01HL122559 (to JEK), 1R15AI113457-01A1 (to MMHK), and K23HD078153-01A1 (to LBH), the Emory University Center for AIDS research (P30AI050409), and the Atlanta Clinical and Translational Sciences Institute (KLR2TR000455, UL1TR000454).

Address correspondence to: Jacob E. Kohlmeier, Department of Microbiology and Immunology, Emory University School of Medicine, Atlanta, Georgia 30322, USA. Phone: 404.727.7023; Email: jkohlmeier@emory.edu.

1. Workowski KA. Centers for disease control and prevention sexually transmitted diseases treatment guidelines. Clin Infect Dis 2015;61(supp1 8):S759-S762.

2. Coker AL. Does physical intimate partner violence affect sexual health? A systematic review. Trauma Violence Abuse. 2007;8(2):149-177.

3. Iijima N, Iwasaki A. Tissue instruction for migration and retention of TRM cells. Trends Immunol. 2015;36(9):556-564.

4. Sheridan BS, Lefrançois L. Regional and mucosal memory T cells. Nat Immunol. 2011;12(6):485-491.

5. Turner DL, Farber DL. Mucosal resident memory CD4 T cells in protection and immunopathology. Front Immunol. 2014;5:331.

6. Amjadi F, Salehi E, Mehdizadeh M, Aflatoonian R. Role of the innate immunity in female reproductive tract. Adv Biomed Res. 2014;3:1. 
7. McMaster SR, Wilson JJ, Wang H, Kohlmeier JE. Airway-resident memory CD8 T cells provide antigen-specific protection against respiratory virus challenge through rapid IFN- $\gamma$ production. J Immunol. 2015;195(1):203-209.

8. Park CO, Kupper TS. The emerging role of resident memory T cells in protective immunity and inflammatory disease. Nat Med 2015;21(7):688-697.

9. Granot T, et al. Dendritic cells display subset and tissue-specific maturation dynamics over human life. Immunity. 2017;46(3):504-515.

10. Mackay LK, et al. The developmental pathway for CD103(+)CD8+ tissue-resident memory T cells of skin. Nat Immunol. 2013;14(12):1294-1301.

11. Swaims-Kohlmeier A, et al. Progesterone levels associate with a novel population of CCR $5^{+} \mathrm{CD} 38^{+} \mathrm{CD} 4 \mathrm{~T}$ cells resident in the genital mucosa with lymphoid trafficking potential. J Immunol. 2016;197(1):368-376.

12. Widom CS, Dutton MA, Czaja SJ, DuMont KA. Development and validation of a new instrument to assess lifetime trauma and victimization history. J Trauma Stress. 2005;18(5):519-531.

13. Koumans EH, et al. The prevalence of bacterial vaginosis in the United States, associations with symptoms, sexual behaviors, and reproductive health. Sex Transm Dis. 2007;34(11):864-869.

14. Segerstrom SC, Miller GE. Psychological stress and the human immune system: a meta-analytic study of 30 years of inquiry. Psychol Bull. 2004;130(4):601-630.

15. Bakdash G, Vogelpoel LT, van Capel TM, Kapsenberg ML, de Jong EC. Retinoic acid primes human dendritic cells to induce gut-homing, IL-10-producing regulatory T cells. Mucosal Immunol. 2015;8(2):265-278.

16. Rodriguez-Garcia M, et al. Dendritic cells from the human female reproductive tract rapidly capture and respond to HIV. Mucosal Immunol. 2017;10(2):531-544.

17. Stary G, et al. VACCINES. A mucosal vaccine against Chlamydia trachomatis generates two waves of protective memory $\mathrm{T}$ cells. Science. 2015;348(6241):aaa8205.

18. Griffith JW, Sokol CL, Luster AD. Chemokines and chemokine receptors: positioning cells for host defense and immunity Annu Rev Immunol. 2014;32:659-702.

19. Sathaliyawala T, et al. Distribution and compartmentalization of human circulating and tissue-resident memory T cell subsets. Immunity. 2013;38(1):187-197.

20. Elwenspoek MMC, Kuehn A, Muller CP, Turner JD. The effects of early life adversity on the immune system. Psychoneuroendocrinology. 2017;82:140-154.

21. Mitchell C, Marrazzo J. Bacterial vaginosis and the cervicovaginal immune response. Am J Reprod Immunol. 2014;71(6):555-563.

22. Wira CR, Rodriguez-Garcia M, Patel MV. The role of sex hormones in immune protection of the female reproductive tract. Nat Rev Immunol. 2015;15(4):217-230.

23. Cohen S, et al. Chronic stress, glucocorticoid receptor resistance, inflammation, and disease risk. Proc Natl Acad Sci U S A. 2012;109(16):5995-5999.

24. Cohen S, Janicki-Deverts D, Miller GE. Psychological stress and disease. JAMA. 2007;298(14):1685-1687.

25. Stewart-Ibarra AM, et al. Psychological distress and Zika, Dengue and Chikungunya symptoms following the 2016 earthquake in Bahía de Caráquez, Ecuador. Int J Environ Res Public Health. 2017;14(12):E1516.

26. Bromley SK, Yan S, Tomura M, Kanagawa O, Luster AD. Recirculating memory T cells are a unique subset of CD4+ T cells with a distinct phenotype and migratory pattern. J Immunol. 2013;190(3):970-976.

27. Milner JJ, Goldrath AW. Transcriptional programming of tissue-resident memory CD8. Curr Opin Immunol. 2018;51:162-169.

28. Hummelen R, et al. Vaginal microbiome and epithelial gene array in post-menopausal women with moderate to severe dryness. PLoS One. 2011;6(11):e26602.

29. Li X, Liu X, Yu S. Psychological stress-derived prolactin modulates occludin expression in vaginal epithelial cells to compromise barrier function. Cell Physiol Biochem. 2015;37(1):153-161.

30. Wira CR, et al. Epithelial cell secretions from the human female reproductive tract inhibit sexually transmitted pathogens and Candida albicans but not Lactobacillus. Mucosal Immunol. 2011;4(3):335-342.

31. Anahtar MN, et al. Cervicovaginal bacteria are a major modulator of host inflammatory responses in the female genital tract. Immunity. 2015;42(5):965-976.

32. Curtis KM, et al. U.S. Medical Eligibility Criteria for Contraceptive Use, 2016. MMWR Recomm Rep. 2016;65(3):1-103.

33. Levenstein S, et al. Development of the Perceived Stress Questionnaire: a new tool for psychosomatic research. J Psychosom Res. 1993;37(1):19-32.

34. Klinger EV, et al. A Community-based study of risk factors for Trichomonas vaginalis infection among women and their male partners in Moshi urban district, northern Tanzania. Sex Transm Dis. 2006;33(12):712-718.

35. Corey L, Huang ML, Selke S, Wald A. Differentiation of herpes simplex virus types 1 and 2 in clinical samples by a real-time taqman PCR assay. J Med Virol. 2005;76(3):350-355. 